applicants. Local anaesthetic training programme being set up via the CESR pathway to help train more anaesthetists. Funded locally by 2 separate NHS trusts

Secondary issues - largest elective waiting list ever in NHS needs more staff trained in surgical specialties. Multiple recruitment rounds for trusts locally to fill non training posts. Wasted time for trainees and HR spent on induction and mandatory statutory training when having to rotate so regularly.

Two Healthcare trusts have collaborated to provide a local training pathway. Previous issues with CESR applications have been the difficult in collecting and providing retrospective evidence of training. By designing a training programme which follows the structure of the GMC guidance for CESR anaesthetic pathway, song with the RCoA curriculum - the typical issues with CESR applications can be avoided by collecting evidence prospectively. The relevant organisations and individuals collaborated through networking and planning towards a shared goal of providing a training programme for future anaesthetists.

This programme provides an opportunity for future leadership development. Leadership is compulsory in traditional training programmes but not necessarily formally taught. By building an autonomous curriculum to match the GMC guidance, leadership and understanding of NHS management will be introduced from the beginning of the programme, and active leadership positions within the trust will be encouraged.

The programme will run over four years across five hospital sites. The time spent at each site will be based upon the trainee's preferences, training needs and service provision. Across all sites there will be a collaborative approach to quality improvement and audits, to maximise output which both the individuals and organisations will benefit

\section{MANAGING MEDICAL EDUCATION DURING THE PANDEMIC-EXPERIENCE OF A PRIVATE MEDICAL SCHOOL IN MALAYSIA}

${ }^{1}$ Ganesh Ramachandran, ${ }^{2}$ Aung Ko Ko Min, ${ }^{3}$ Munandy Alagar. 'Taylor's University Malaysia; ${ }^{2}$ MAHSA University Malaysia; ${ }^{3}$ UCSI University Malaysia

\subsection{6/leader-2021-FMLM.40}

This work was undertaken in a private medical school in Malaysia, it looks at interventions undertaken due to the COVID-19 pandemic and impact on students. The pandemic disrupted the accepted norms for delivery in the faculty, requiring quick changes to ensure uninterrupted teaching, learning and assessment for students. The problems were instant conversion to on online format with the use of simulation and recorded practical sessions for theory and practical sessions respectively. The other major issue was to educate staff and students on this matter and update delivery platforms.

The immediate solutions

- Use the platforms immediately available.

- Determine a minimum level of competency required to deliver the curriculum.

- Engage stakeholders.

- Ensure faculty development

- Adhere to regulatory frameworks.
This was communicated to all stakeholders and lines of communication formed to engage and mentor students through the period. Feedback on the sessions were sought. Students were constantly reminded this was non-punitive. Objective measures were to compare assessment outcomes of high stakes examinations from the previous years. Questionnaire surveys were used for feedback. Pass rates with previous cohorts were compared. The preliminary result indicates general satisfaction with teaching and learning, preference of the online platform for formative assessment and a comparable performance in all high stakes examinations.

Impact

- The online platform is an acceptable for both instructors and students.

- Formative assessment was preferred on this platform.

- Delivery of the curriculum was continued without disruption if you started with what was available and then developed capabilities

- Communication was important.

Disruption forces innovation and movement out of comfort zones, medical education has largely depended on face-to-face delivery. The pandemic has allowed more innovative delivery.

\section{MAXIMISING ENDOSCOPY TRAINING DURING THE COVID-19 RECOVERY PHASE AT ST MARKS HOSPITAL}

A Shalabi, SM Ha, L Dyall, D Chaterjee, S Pomfret, A Haycock. The Wolfson Unit for Endoscopy, St Mark's Hospital, London, UK

\subsection{6/leader-2021-FMLM.41}

Aims Our unit has 24 gastroenterology and surgical registrars training in endoscopy. Access to endoscopy at St Mark's Hospital has historically been a challenge due to the number of trainees requiring training lists with the demand on the unit to perform tertiary centre complex cases.

The COVID-19 pandemic has further impacted the situation. Trainees have been struggling to meet targets (procedure numbers and exposure to therapy) set out by Joint Advisory Group on GI Endoscopy (JAG) to become competent endoscopists.

Methods Internal teaching courses were run to increase the number of trainers.

An agreement was reached that all lists would be open to trainees without reducing the number of cases. All available endoscopy lists were scrutinised to establish suitability for training. A senior endoscopy trainee acted as the named allocator contacting the other trainees to ascertain their current training needs and weekly commitments. This information was used to allocate appropriate lists to each trainee. A timetable was disseminated to all trainees and trainers every 2 weeks.

The JAG endoscopy training portfolio was used to assess the number of procedures completed by trainees, and a satisfaction survey was conducted pre- and post-intervention.

Results Following our intervention, 10 further trainers were made available. The number of lists attended by trainees increased by 74\%(171 between September 2020 and February 2021 versus 298 between March 2021 and June 2021), gastroscopies performed by 57\% (267 vs 419) and colonoscopies by $111.4 \%$ (175 vs 370$)$. 
Satisfaction with access to endoscopy training rose from $50 \%$ to $100 \%$ and satisfaction with communication increased from $66.7 \%$ to $100 \%$.

Conclusion Satisfaction amongst trainees and up-skilling in endoscopy has improved significantly with our intervention.

Our model was devised by trainees for trainees. This could be implemented across all trusts and future regional academies to improve access to endoscopy training.

\section{MEASURING DISRUPTION OF UK GENERAL PRACTICE BY DIGITAL FIRST PRIMARY CARE: A CASE STUDY EXAMINING THE IMPACT OF BABYLON GP AT HAND IN LONDON}

Saqib Zakaria Latif, Jonathan Turnbull-Ross, Hui Wang, Sudip Chowdhury. Alliance Manchester Business School, UK

\subsection{6/leader-2021-FMLM.42}

Background General Practice is central to the NHS but remains difficult to access for the working well. It has resisted organisational change leaving it resistant to technology and vulnerable to competition. Privately funded Babylon GP at Hand (BGPaH) can disrupt through a value proposition (access) whilst undermining core funding.

Aim It is important to analyse the impact that Babylon has made in attracting London based patients. In 2017 Babylon created a joint venture with a London based practice resulting in list growth to the 5th largest in the UK. The study examines factors that make General Practice vulnerable to digital transformation.

Design and Setting Adopting a qualitative case-study approach we assessed GP awareness of technological disruption and their willingness to utilise scale-economics through organisational development. The setting of our research was London. Quantitatively, we investigated patient-flow within Hammersmith and Fulham CCG.

Method We utilised a mixed-methods approach utilising semistructured interviews and analysis of public data. Six GPs and two senior health managers were interviewed $(n=8)$ and then analysed using thematic analysis. We also accessed the NHS Business Services Authority Database to compare numbers for each practice in Hammersmith and Fulham on November 2017 and July 2020.

Results Five broad themes were identified including GP Business Ideation, GP Organisational Development, Economies of Scale, COVID-19, and the NHS. The quantitative aspects of the study demonstrated a statistically significant increase in patient number by BGPaH.

Conclusion GPs exhibited an awareness of business threat from $\mathrm{BGPaH}$ but this did not translate to organic organisational change. Factors included the pandemic and the use of technology as well as the emergence of PCNs. There was evidence of successful use of economies of scale by a GP-owned Federation. We could not quantitatively prove that $\mathrm{BGPaH}$ had disrupted primary care.

\section{A NEW STUDENT LED LEADERSHIP PROGRAMME AT WARWICK MEDICAL SCHOOL}

Julia Jones, Charlotte Caroff, Anne-Marie Chilton. Warwick Medical School

10.1136/leader-2021-FMLM.43
This year marks the completion of the inaugural Warwick Medical School Leadership programme. The programme was set up to meet student interest and the need for an effective medical student leadership curriculum highlighted by the UK General Medical Council, the NHS Leadership Academy and The King's Fund.

The programme's aim is to help produce valued future leaders of the NHS. It focuses on creating an engaging and inclusive curriculum for time poor students from a variety of backgrounds.

To achieve this aim, we needed to not only give our students a foundation in leadership skills but empower and inspire them to continue developing these skills outside the course. We devised 5 components to the course, not only to teach the basics but also to support students in building a network of resources and leadership mentors. Students were given tasks to use these tools to understand their benefits for their own ongoing projects .

Within each of the course's assessments students showed an understanding and enthusiasm for the course. Comparing their initial 'project plan' assessment and the final summative reflection, all students demonstrated a developing understanding of leadership roles, greater personal insight and the desire and confidence to develop as a leader. Within the summative reflection, all students stated the course had a positive impact on their current leadership roles

Within the end of course feedback, when asked what they found helpful about the course, students described developing a support network of peers and mentors, interesting and thought provoking seminars and a new confidence to take on new leadership positions and projects.

We are very pleased with how the first year has been received by the students. We recommend other universities encourage similar courses to run. The course has so far proven self-sustaining, with its students developing the skills required to continue its development and the enthusiasm to take over its leadership.

\section{NATIONAL LEARNER ASSEMBLY FOR EQUALITY, DIVERSITY AND INCLUSION}

Gregory FW Stamp, Raees Lunat, Vidushi Golash. Health Education UK

\subsection{6/leader-2021-FMLM.44}

HEE's Summer 2020 call for evidence answered by over 400 trainees the aftermath of Black Lives Matter. Analysis of these responses showed system-wide challenges that trainees face. Those of minority backgrounds and International Medical Graduates are acutely aware of differential attainment and a lack of representative role-modelling. One stand-out issue was difficulties in having trainee views and experiences represented to senior leaders of HEE in open dialogue. This Assembly aims to improve this situation.

Our inaugural event was 26th May 2021. It was attended by Dr Navina Evans and Prof. Namita Kumar, outlining their experiences of the importance of diverse leadership.

An interactive roundtable discussion with over 70 participants followed. Trainees' personal stories were heard and reflected upon. A range of themes emerged, including EDI in recruitment processes, clinical supervision and flexible training options. Some individuals shared experiences of differential attainment, systemic racism and undermining behaviours. 